\title{
Enterovirus infections in Singaporean children: an assessment of neurological manifestations and clinical outcomes
}

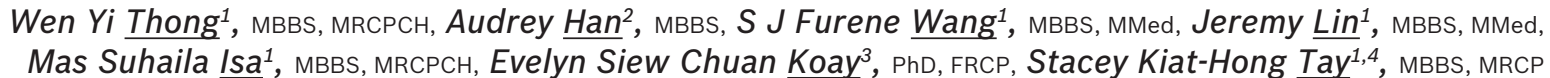

INTRODUCTION Enterovirus infections in childhood can be associated with significant neurological morbidity. This study aimed to describe the prevalence and range of neurological manifestations, determine the clinical characteristics and assess differences in clinical outcomes for Singaporean children diagnosed with enterovirus infections.

METHODS In this single-centre, case-control study, clinical data was collected retrospectively from patients admitted to National University Hospital, Singapore, from August 2007 to October 2011 and diagnosed with enterovirus infection, based on the enterovirus polymerase chain reaction test, or cultures from throat and rectal swabs or cerebrospinal fluid samples. The occurrence of neurological manifestations was reviewed and clinical outcomes were assessed.

RESULTS A total of 48 patients (age range: six days -17.8 years) were included in the study. Neurological manifestations were seen in $75.0 \%$ of patients, $63.9 \%$ of whom presented with aseptic meningitis. Other neurological manifestations included encephalitis, acute cerebellitis, transverse myelitis and autonomic dysfunction. The incidence of neurological manifestations was significantly higher in patients aged $>1$ year as compared to younger patients $(p=0.043)$. In patients without neurological manifestations, a significantly higher proportion presented with hand, foot and mouth disease and poor feeding. Long-term neurological sequelae were seen in $16.7 \%$ of patients with neurological manifestations.

CONCLUSION A wide spectrum of neurological manifestations resulting in a relatively low incidence of long-term neurological sequelae was observed in our study of Singaporean children with enterovirus infections. As some of these neurological morbidities were severe, careful evaluation of children with neurological involvement is therefore necessary.

Keywords: encephalitis, enterovirus infection, hand foot and mouth disease, meningitis

\section{INTRODUCTION}

Enterovirus infections have been recognised as one of the more common childhood infections. Available literature has focused mainly on hand, foot and mouth disease (HFMD), herpangina and enterovirus 71 (EV71) infections. Numerous outbreaks of HFMD have been reported in Asia, including in Singapore, ${ }^{(1)}$ Malaysia, ${ }^{(2)}$ Taiwan, ${ }^{(3)}$ Korea $_{,}^{(4)}$ Vietnam ${ }^{(5)}$ and China, ${ }^{(6)}$ indicating that this is a significant problem in the region. Neurological manifestations associated with enterovirus infections include aseptic meningitis, encephalitis, polio-like syndrome ${ }^{(7)}$ and myoclonic jerks. ${ }^{(8)}$ In 2008, Singapore experienced one of its largest HFMD outbreaks with 29,686 cases. ${ }^{(9)}$ In a Singaporean study involving 43 children with HFMD during the 2008 outbreak, the most prevalent enterovirus serotypes were coxsackievirus A6 (CA6), coxsackievirus A10 (CA10) and EV71, with CA6 and CA10 accounting for $23.5 \%$ and $11.8 \%$ of all HFMD cases, respectively, and EV71 accounting for $21.6 \%$ of the patients. ${ }^{(9)}$ In a recent study involving children hospitalised for HFMD in China, EV71 and coxsackievirus A16 (CA16) accounted for $21 \%$ and $16.1 \%$ of patients, respectively. ${ }^{(10)}$ Another recent study from Korea showed a predominance of EV71 (72.6\%) isolation in children admitted for HFMD and herpangina. ${ }^{(7)}$ All these studies reflect a varying distribution of enterovirus serotypes resulting in disease. EV71 is known to result in more neurological morbidity compared to other serotypes, including CA16 ${ }^{(10)}$ and coxsackievirus A2. ${ }^{(11)}$ Previous studies have shown that several factors, such as the patient's age, ${ }^{(12)}$ presence of HFMD rash, duration of fever, peak body temperature and presence of neurological signs, ${ }^{(7)}$ were associated with neurological manifestations in patients with HFMD and EV71 infections.

While there have been several previous studies on enterovirus infections in Singaporean children, none of these studies focused on the neurological manifestations associated with the disease. . $1,9,13-15)^{2}$ This study aimed to describe the prevalence and range of neurological manifestations in Singaporean children diagnosed with enterovirus infections. It also aimed to determine the possible clinical characteristics associated with the development of neurological manifestations in these children and to assess differences in clinical outcomes for patients with and without neurological manifestations.

\section{METHODS}

This single-centre, retrospective, case-control study included all patients - from newborn infants to children aged 18 years - who required admission to the paediatric unit at National University Hospital (NUH), Singapore, after presenting to the outpatient

${ }^{1}$ Khoo Teck Puat-National University Children's Medical Institute, National University Health System, ${ }^{2}$ Yong Loo Lin School of Medicine, National University of Singapore, ${ }^{3}$ Laboratory Medicine, Molecular Diagnosis Centre, National University Health System, ${ }^{4}$ Department of Paediatrics, Yong Loo Lin School of Medicine, National University of Singapore, Singapore Correspondence: Dr Wen Yi Thong, Consultant, Khoo Teck Puat-National University Children's Medical Institute, National University Health System, Level 12 NUHS Tower Block 1 E, Lower Kent Ridge Road, Singapore 119228. wen_yi_thong@nuhs.edu.sg 
clinic or the children's emergency department with a clinical diagnosis of enterovirus infection between August 2007 and October 2011, and who were subsequently confirmed to have an enterovirus infection based on a positive enterovirus polymerase chain reaction (PCR) test or virus isolation during admission.

Rectal and throat swabs were sent for enterovirus studies based on the clinical diagnosis of HFMD. Cerebrospinal fluid (CSF) studies were done in addition to rectal and throat swabs if the patient presented with neurological symptoms. Referrals were made to the paediatric neurology team for all patients with suspected neurological manifestations. Neurological manifestations were documented in the clinical notes after a clinical assessment by a paediatric neurology consultant. This ensured that the neurological assessment was reliable and also helped to minimise interobserver variability.

Patients were identified retrospectively through a positive enterovirus PCR test or virus isolation records maintained in the Department of Laboratory Medicine, NUH. A diagnosis of enterovirus infection was made if there was a positive enterovirus PCR test, or virus culture from CSF, throat or rectal swab samples. In addition, EV71 serotyping was carried out for patients who were positive for enterovirus PCR or viral culture.

Data was collected retrospectively from the medical records of these identified patients and reviewed; it included demographic data, clinical presentations and examination findings, presence of a pre-existing neurological disorder, occurrence of neurological manifestations, laboratory investigations, neuroimaging results, types of treatment administered and clinical outcomes. Case records were reviewed independently by a medical student and an experienced clinician (paediatrician) to ensure accuracy of data collection.

HFMD was defined as having vesiculopapular rashes over the hands, soles or buttocks, and mouth ulcers, while herpangina was defined as having oral ulcers without any skin rash. The development of neurological manifestations was defined as a diagnosis of any neurological disorder associated with the diagnosis of enterovirus infection. The presence of a pre-existing neurological disorder included a diagnosis of any neurological disorder that had been made prior to the presentation for enterovirus infection and that was included in the background history of the patient. Aseptic meningitis was defined as having clinical signs or symptoms of meningitis with pleocytosis (leucocyte $>22$ cells $/ \mathrm{mm}^{3}$ in neonates, $>15$ cells $/ \mathrm{mm}^{3}$ in infants aged $1-2$ months, or $>5$ cells $/ \mathrm{mm}^{3}$ in patients aged $>2$ months; and red blood cell $[\mathrm{RBC}]<1,000 / \mathrm{mm}^{3}$ ) and a negative bacterial culture of the CSF samples obtained from lumbar puncture. ${ }^{(16,17)}$ Encephalitis was defined as having an acute brain infection associated with alteration in the level of consciousness. ${ }^{(18)}$ Poor feeding was defined as taking less than half of the normal oral intake or requiring intravenous hydration.

Data was analysed using SPSS version 10.0 (SPSS Inc, Chicago, IL, USA). Normally distributed data was compared using Student's $t$-test; data that was not normally distributed was compared using Mann-Whitney $U$ test. Categorical data was tested using Pearson's chi-square test or Fisher's exact test. A p-value $<0.05$ was considered to be statistically significant.

\section{RESULTS}

A total of 48 patients were diagnosed with enterovirus infection during the study period based on a positive enterovirus PCR test, or culture reports of CSF, throat or rectal swab samples. The neurological manifestations seen in these patients are shown in Table I and Fig. 1.36 (75.0\%) of the patients developed some form of neurological manifestation, 23 (63.9\%) of whom presented

Table I. Neurological manifestations in patients with enterovirus infection $(n=36)$.

\begin{tabular}{lc}
\hline Manifestation & No. (\%) \\
\hline Aseptic meningitis & $23(63.9)$ \\
Seizures & $8(22.2)$ \\
Encephalitis & $6(16.7)$ \\
Autonomic dysfunction & $1(2.8)$ \\
Transverse myelitis & $1(2.8)$ \\
Acute cerebellitis & $1(2.8)$ \\
Paralysis & $0(0)$ \\
Mortality & $0(0)$ \\
\hline
\end{tabular}

Total no. adds up to $>36$ as some patients had $>1$ manifestation.

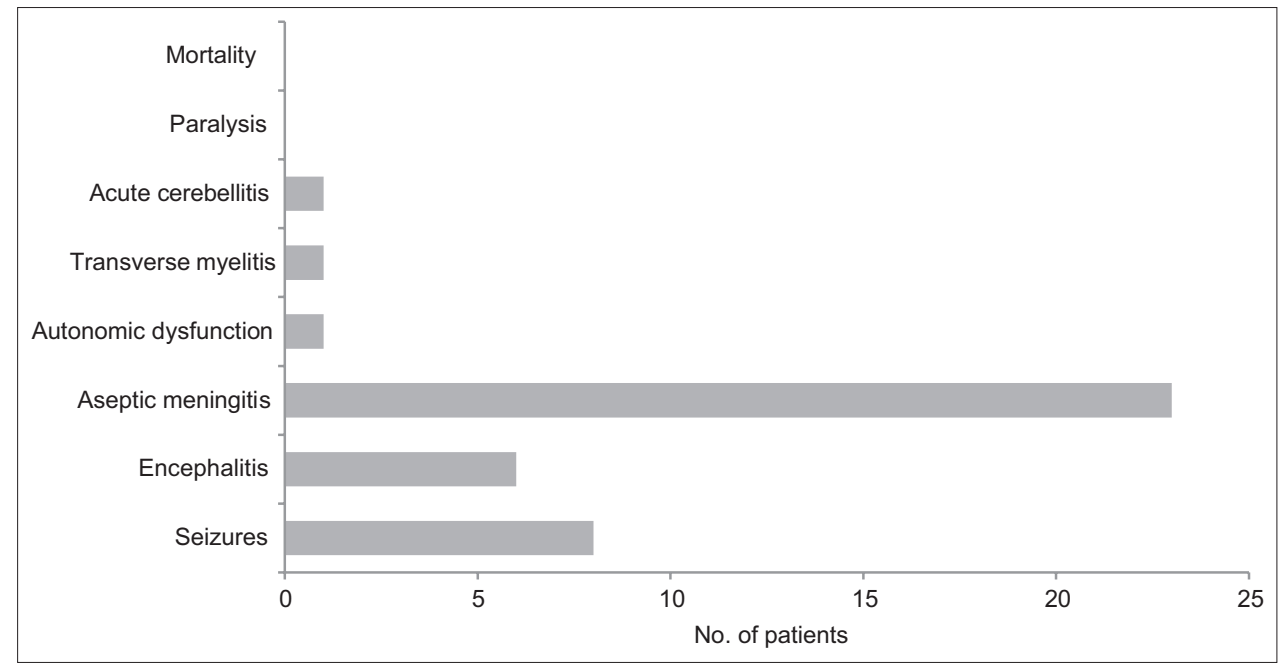

Fig. 1 Graph shows the range of neurological manifestations in the patients $(n=36)$. 
with aseptic meningitis, $6(16.7 \%)$ presented with encephalitis, and $8(22.2 \%)$ developed seizures during the acute illness. Other neurological manifestations included acute cerebellitis $(n=1)$, transverse myelitis $(n=1)$ and autonomic dysfunction $(n=1)$. There were no clinical presentations with polio-like syndrome and no mortalities were recorded.

Non-neurological presentations included HFMD, herpangina, gastrointestinal symptoms and acute liver dysfunction. There was a higher proportion of patients with HFMD among patients who did not develop neurological manifestations compared to those who developed neurological manifestations (33.3\% vs. 2.8\%; $p=0.011$ ). There was no difference in the prevalence of other non-neurological presentations among patients with and without neurological manifestations.

The demographic data of the patients, including age range, is shown in Table II. There were 31 (64.6\%) boys and 17 (35.4\%) girls, with a median age of 4.3 months (range 6 days- 17.8 years). The peak incidence of enterovirus infection was seen in patients aged $\leq 1$ year $(n=28,58.3 \%$ ) (Fig. 2). There were 21 (43.8\%) Chinese, 16 (33.3\%) Malay and 4 (8.3\%) Indian patients, with the rest ( $n=7,14.6 \%$ ) belonging to other ethnicities.

Of the 28 infants (patients aged $\leq 1$ year) with enterovirus infection, 18 (64.3\%) developed neurological manifestations. In comparison, 18 out of $20(90.0 \%)$ patients aged $>1$ year presented with neurological manifestations. There was a significantly higher incidence of neurological manifestations in patients aged $>1$ year $(p=0.043)$. There was no significant difference in gender or racial distribution between patients with and without neurological manifestations. Among the 48 patients in the study cohort, 8 (16.7\%) were known to have pre-existing neurological disorders. This included a history of febrile seizures, migraine, chronic motor tics and cerebral palsy. There was no significant difference in the incidence of a pre-existing neurological disorder in patients with and without neurological manifestations.

The clinical manifestations of the patients are summarised in Table III. The most common presenting symptoms were fever $(87.5 \%)$, lethargy and/or irritability $(41.7 \%)$, and poor oral intake $(35.4 \%)$. Of these presenting symptoms, only poor oral intake was significantly associated with patients without neurological manifestations; poor feeding was present in $75.0 \%$ of patients who did not develop neurological manifestations, but in only $22.2 \%$ of patients who developed neurological manifestations $(p=0.002)$. Headache, neck ache and/or photophobia, seizures and altered mental state were only present in patients who developed neurological manifestations. The median time from the onset of symptoms (such as fever) to hospital presentation was not significantly different between patients with and without neurological manifestations.

Table II. Demographics of patients with and without neurological manifestations.

\begin{tabular}{|c|c|c|c|c|}
\hline \multirow[t]{2}{*}{ Variable } & \multicolumn{3}{|c|}{ No. (\%) } & \multirow[t]{2}{*}{ p-value } \\
\hline & Total $(n=48)$ & With neurological manifestation $(n=36)$ & Without neurological manifestation $(n=12)$ & \\
\hline Age (mth)* & $4.3(0.02-213.00)$ & $17.5(0.20-213.00)$ & $2.8(0.02-13.00)$ & 0.057 \\
\hline Ethnicity & & & & 0.524 \\
\hline Chinese & $21(43.8)$ & $15(41.7)$ & $6(50.0)$ & \\
\hline Malay & $16(33.3)$ & $11(30.6)$ & $5(41.7)$ & \\
\hline Indian & $4(8.3)$ & $4(11.1)$ & $0(0)$ & \\
\hline Others & 7 (14.6) & $6(16.7)$ & $1(8.3)$ & \\
\hline
\end{tabular}

*Data presented as median (interquartile range).

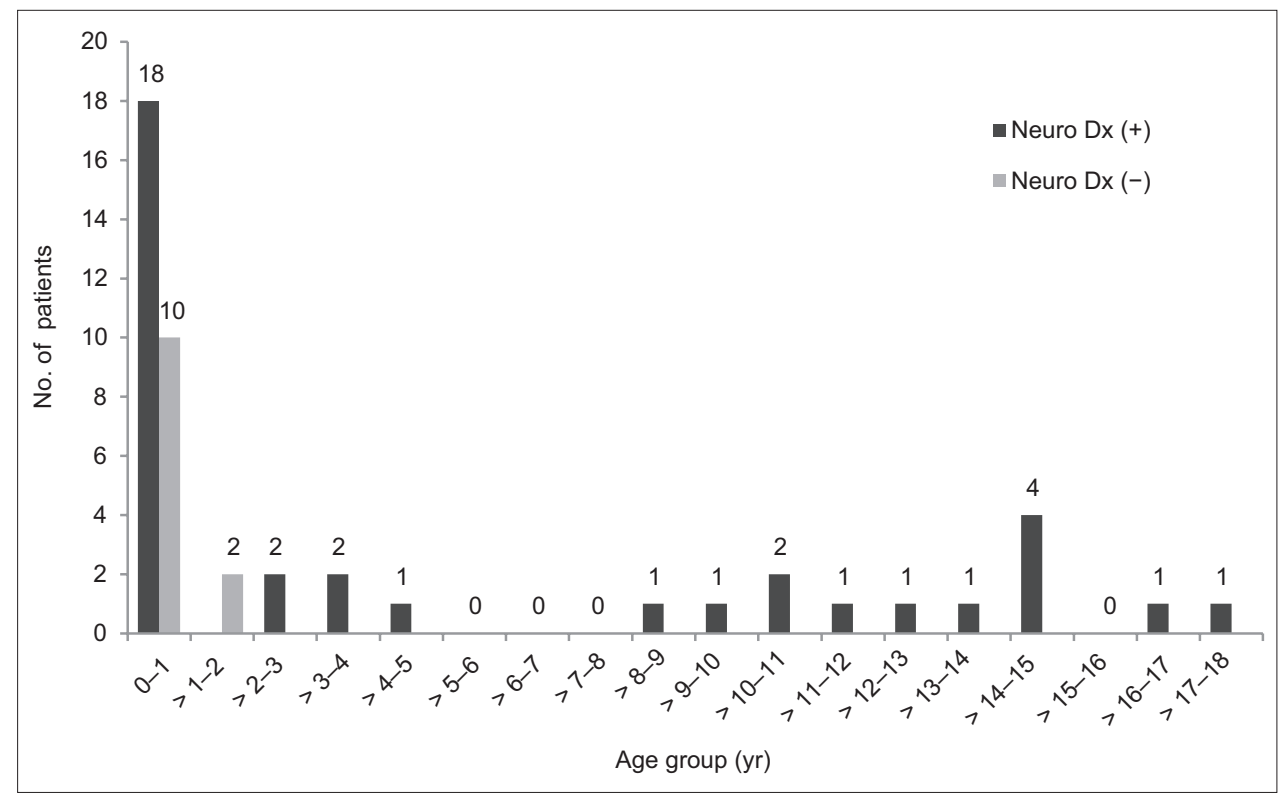

Fig. 2 Graph shows the age distribution of patients $(n=36)$ with enterovirus infection. Neuro Dx: neurological diagnosis. 
Table III. Clinical manifestations of patients with and without neurological manifestations.

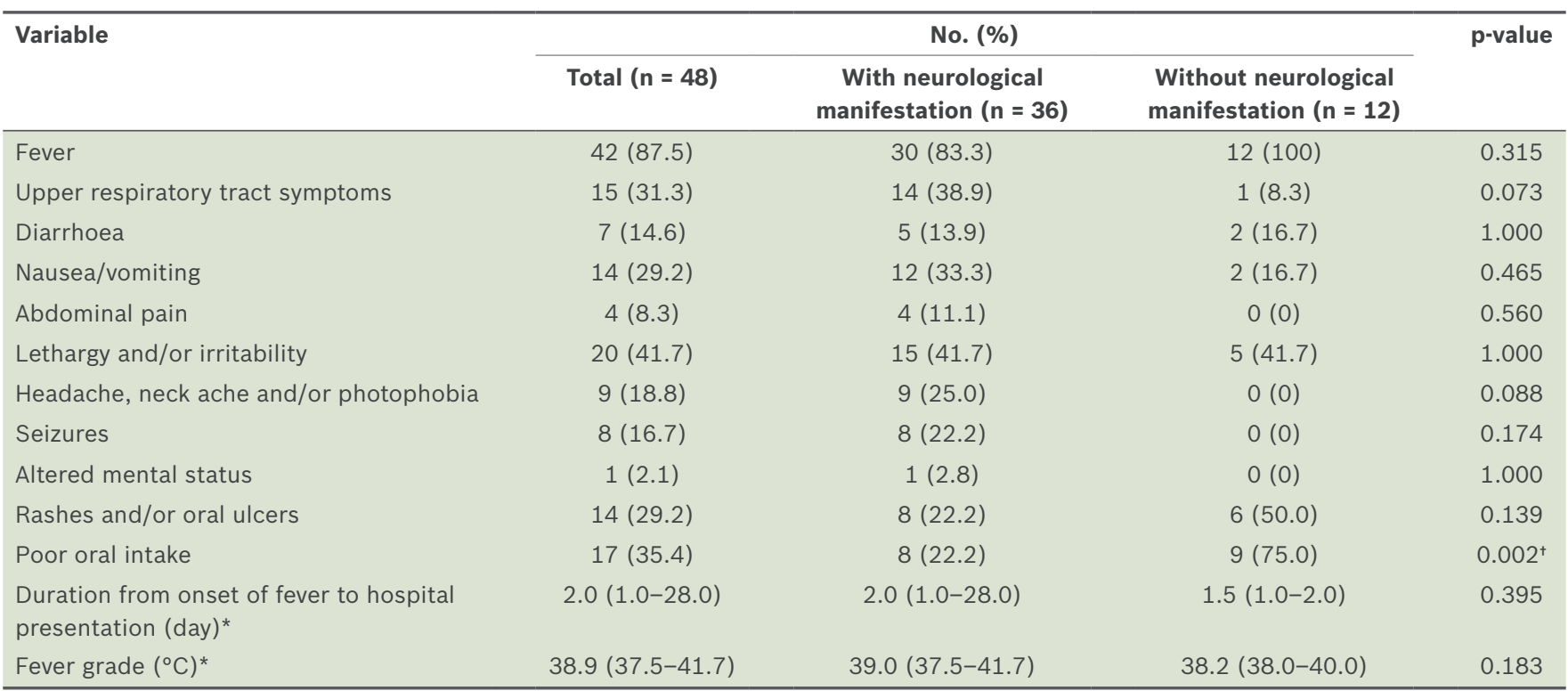

*Data was calculated from a non-normal distribution and presented as median (interquartile range). $+\mathrm{p}<0.05$ was statistically significant.

Abnormal physical findings in the patients included abnormal tone (hypotonia and hypertonia) and reflexes (hyperreflexia and hyporeflexia), altered mental status, cerebellar signs and signs of meningism. All abnormal neurological findings were found in patients who developed neurological manifestations. Signs of meningism (such as nuchal rigidity, Kernig's sign and photophobia) were only present in patients diagnosed with aseptic meningitis. However, $69.6 \%$ of patients with aseptic meningitis did not display any signs of meningism.

Enterovirus was most commonly isolated successfully from rectal swabs $(93.3 \%)$, followed by throat swabs $(80.0 \%)$ and CSF samples $(78.8 \%)$. In patients presenting with neurological manifestations, $90.0 \%$ of rectal swabs, $73.7 \%$ of throat swabs and $85.7 \%$ of CSF samples tested positive for enterovirus. In comparison, in patients without neurological manifestations, $100 \%$ of rectal and throat swabs and $40.0 \%$ of CSF samples tested positive for enterovirus. EV71 serotype testing was carried out in 23 of the 48 patients and EV71 was isolated from 13.0\% of CSF, throat or rectal swab samples tested. Only $5.3 \%$ of patients with neurological manifestations tested positive for EV71, while $50.0 \%$ of patients without neurological manifestations tested positive for EV71. There was no significant difference in the rates of EV71 isolation in patients with and without neurological manifestations. Of the three patients who tested positive for EV71 serotype, only 1 (33.3\%) patient developed neurological manifestations.

The findings of laboratory investigations for these patients are summarised in Table IV. Patients without neurological manifestations were noted to have a significantly higher serum C-reactive protein (CRP) level $(p=0.013)$. The median serum CRP level in patients without neurological manifestations was 17 (range 10-66) mg/L compared to a median serum CRP level of 0 (range 0-126) $\mathrm{mg} / \mathrm{L}$ in patients with neurological manifestations (normal range $0-10 \mathrm{mg} / \mathrm{L}$ ). No other significant differences with regard to blood investigations (e.g. blood glucose, total white blood cell count, platelet count and haemoglobin level) were noted between patients with and without neurological manifestations

CSF pleocytosis was only detected in patients who developed neurological manifestations $(p=0.006)$. Significantly higher CSF leucocyte counts and CSF RBC counts were noted in patients with neurological manifestations. The median CSF leucocyte count was 90 (range 0-531) cells $/ \mathrm{mm}^{3}$ in patients with neurological manifestations and 5 (range $0-10$ ) cells $/ \mathrm{mm}^{3}$ in those without neurological manifestations $(p=0.021)$. Similarly, the median CSF RBC count was 10 (range $0-8,370$ ) cells $/ \mathrm{mm}^{3}$ in patients with neurological manifestations and 0 (range $0-8$ ) cells $/ \mathrm{mm}^{3}$ in those without neurological manifestations $(p=0.015)$.

Neuroimaging and electroencephalogram (EEG) were done for patients with neurological manifestations based on clinical indications. EEG was performed for all patients who presented with seizures. The EEG protocol of the study centre mandated that the EEG be done in the acute setting if there were concerns about subclinical seizures or non-convulsive status epilepticus. If otherwise, EEG would be done 2-3 weeks after the acute seizures. Of the seven patients who were investigated with EEG due to seizures, five showed EEG abnormalities. All neuroimaging was done during admission for the acute infection and $40 \%$ of imaging performed in patients who developed neurological manifestations was found to be abnormal. Based on magnetic resonance (MR) imaging, one patient demonstrated a hyperintense, non-enhancing lesion in the cervical spinal cord consistent with the clinical diagnosis of transverse myelitis, two patients had subcortical white matter changes bilaterally, and another patient was found to have hyperintensities in the basal ganglia and brainstem. These patients did not have any pre-existing neurological disorders prior to the acute presentation. One patient with a background history of cerebral palsy had areas of restricted diffusion involving the corpus callosum and bilateral globus pallidus on previous MR imaging 
Table IV. Findings of laboratory investigations for patients with and without neurological manifestations.

\begin{tabular}{|c|c|c|c|c|}
\hline \multirow[t]{2}{*}{ Variable } & \multicolumn{3}{|c|}{ No. (\%) } & \multirow[t]{2}{*}{ p-value } \\
\hline & Total $(n=48)$ & $\begin{array}{c}\text { With neurological } \\
\text { manifestation }(n=36)\end{array}$ & $\begin{array}{l}\text { Without neurological } \\
\text { manifestation }(n=12)\end{array}$ & \\
\hline Red blood cells $\left(\times 10^{9} / \mathrm{L}\right)^{*}$ & $4.43(3.08-5.72)$ & $4.48(3.13-5.72)$ & $4.27(3.08-5.32)$ & 0.395 \\
\hline Platelets $\left(\times 10^{9} / \mathrm{L}\right)^{*}$ & $362(12-727)$ & $380(169-727)$ & $298(12-551)$ & 0.123 \\
\hline White blood cells $\left(\times 10^{9} / \mathrm{L}\right)^{*}$ & $11.67(4.63-24.71)$ & $11.31(4.62-21.71)$ & $12.88(8.71-18.77)$ & 0.337 \\
\hline Blood glucose $(\mathrm{g} / \mathrm{dL})^{*}$ & $5.4(3.3-6.0)$ & $5.4(3.3-6.0)$ & $5.3(4.5-5.7)$ & 0.943 \\
\hline Serum C-reactive protein $(\mathrm{mg} / \mathrm{L})^{*}$ & $6(0-126)$ & $0(0-126)$ & $17(10-66)$ & $0.013^{+}$ \\
\hline CSF red blood cells $\left(/ \mathrm{mm}^{3}\right)^{*}$ & $8(0-8,370)$ & $10(0-8,370)$ & $0(0-8)$ & $0.015^{+}$ \\
\hline CSF white blood cells $\left(/ \mathrm{mm}^{3}\right)^{*}$ & $64(0-531)$ & $90(0-531)$ & $5(0-10)$ & $0.021^{+}$ \\
\hline \multicolumn{5}{|l|}{ Imaging/other modalities } \\
\hline Brain $\mathrm{CT} / \mathrm{MR}$ imaging & $11(22.9)$ & $10(27.8)$ & $1(8.3)$ & 0.455 \\
\hline Normal & $6(54.5)$ & $6(60.0)$ & $0(0)$ & \\
\hline Abnormal & $5(45.5)$ & $4(40.0)$ & $1(100)$ & \\
\hline EEG & $7(14.6)$ & $6(16.7)$ & $1(8.3)$ & 1.000 \\
\hline Normal & $2(28.6)$ & $2(33.3)$ & $0(0)$ & \\
\hline Abnormal & 5 (71.4) & $4(66.7)$ & $1(100)$ & \\
\hline
\end{tabular}

*Data presented as median (interquartile range). ${ }^{+} \mathrm{p}<0.05$ was statistically significant. CSF: cerebrospinal fluid; CT: computed tomography; EEG: electroencephalogram; MR: magnetic resonance

of the brain, performed prior to the acute episode of enterovirus infection. This patient did not develop any new neurological manifestations associated with the acute enterovirus infection. She subsequently underwent neuroimaging and EEG based on clinical indications, and repeat MR imaging did not reveal any new changes.

There were $8(16.7 \%)$ patients who were treated with antiviral medication (intravenous acyclovir), while 34 (70.8\%) patients had been empirically treated with antibiotics prior to the diagnosis of enterovirus infection. Out of the 36 patients with neurological manifestations, $6(16.7 \%)$ were treated with antiepileptic drugs. Only two of these six patients required long-term antiepileptic medications for more than six months' duration. There was no significant difference in treatment between patients with and without neurological manifestations.

Clinical outcomes assessed included long-term neurological sequelae; loss of independence of activities of daily living (ADL) in patients who were able to perform self-care before the onset of enterovirus infection; ventilator dependence following hospital discharge; and total duration of hospitalisation (Table V). Among all patients, the median time to post-morbid assessment of longterm neurological sequelae in this study was eight months. Of the 48 patients recruited for this study, $6(12.5 \%)$ were lost to follow-up. The clinical assessment for long-term neurological outcomes was carried out by a paediatrician in the outpatient clinic. Follow-up for these patients was based on the clinical decision of the paediatrician in charge after the follow-up assessment. Children who continued to demonstrate postdischarge neurological sequelae were followed up regularly as clinically indicated.

Of the 36 patients who developed neurological manifestations during the enterovirus infection, $6(16.7 \%)$ patients demonstrated long-term neurological sequelae. Long-term neurological sequelae that were observed included epilepsy $(n=1)$, chronic myoclonic
Table V. Clinical outcomes of patients with neurological manifestations $(n=36)$.

\begin{tabular}{lc}
\hline Outcome & No. (\%) \\
\hline Long-term neurological morbidity & $6(16.7)$ \\
Need for long-term antiepileptic drug use & $2(5.6)$ \\
Epilepsy & $1(2.8)$ \\
Chronic myoclonic jerks & $1(2.8)$ \\
Behavioural changes/cognitive decline & $1(2.8)$ \\
Developmental delay & $2(5.6)$ \\
Autonomic dysfunction & $1(2.8)$ \\
Loss of ADL independence & 0 \\
Ventilator dependence & 0 \\
Time to post-morbid assessment (mth)* & $7.42(0.07-15)$ \\
Length of hospital stay (day)* & $4.92(1-35)$ \\
\hline
\end{tabular}

*Data presented as median (interquartile range). ADL: activities of daily living

jerks $(n=1)$, behavioural changes or cognitive decline $(n=1)$, developmental delay $(n=2)$ and autonomic dysfunction $(n=1)$. In the patient who had persistent autonomic dysfunction with mydriasis, widespread loss of sweating and bladder dysfunction was seen. All the long-term neurological sequelae observed in our study developed after the onset of enterovirus infection and occurred in patients without pre-existing neurological disorders. None of these patients were ventilator-dependent after hospital discharge.

The median duration of hospitalisation was 4.8 (range 1-35) days. Of the four patients who were hospitalised beyond 14 days, three patients required management of comorbidities unrelated to the enterovirus infection and one patient required extended caregiver training for home nasogastric tube feeding. There was no significant difference in time to post-morbid assessment between patients with and without neurological manifestations. There was also no significant difference in the duration of hospitalisation between the two groups of patients. 


\section{DISCUSSION}

While several previous studies examined Singaporean children with enterovirus infections, ${ }^{(1,9,13-15)}$ none of them focused on the neurological manifestations associated with the disease. This is of clinical concern, as enterovirus infection is one of the more common childhood infections and the neurological manifestations arising from enterovirus infections can lead to long-term neurological sequelae.

In this study, we found that $75.0 \%$ of patients with documented enterovirus infections presented with some form of neurological manifestation. This result is reflective of a population of patients who are diagnosed with enterovirus infection and require hospital admission. Two-thirds of these patients presented with aseptic meningitis, while the rest presented with encephalitis, acute cerebellitis, transverse myelitis and autonomic dysfunction, indicative of a broad spectrum of neurological morbidities associated with enterovirus infections in Singaporean children.

We detected unusual neurological disease presentations, including acute cerebellitis and autonomic dysfunction. A patient with acute cerebellitis developed signs of cerebellar dysfunction two days after the onset of symptoms of infection. Another patient presented with mydriasis, widespread loss of sweating and bladder dysfunction during the acute infection. These unusual neurological disease presentations should be recognised as part of the wide spectrum of neurological manifestations associated with enterovirus infections.

In a study from Taiwan, Wang et al found that with decreasing age of onset of the enterovirus infection, there was an increase in disease severity with regard to central nervous system complications. ${ }^{(12)}$ Only $13.4 \%$ of children were aged below one year in their study. In comparison, we found that the peak incidence of enterovirus infection in our local population was before one year of age. We also noted that there was a higher incidence of neurological manifestations in children aged over one year. The reasons for the above observations, based on our study, are unclear. It may be a reflection of the varying distribution of enterovirus serotypes, resulting in differences in disease presentation. Future studies involving non-EV71 serotyping for children with enterovirus infections may provide us with more information.

In our study, headache, photophobia, seizures, presence of abnormal physical signs and CSF pleocytosis were only found in patients who developed neurological manifestations. The presence of these symptoms and signs could indicate the development of neurological complications in a child diagnosed with enterovirus infection. This is not unexpected, as a Korean study by Kim et al showed that headache and neurological signs were significantly associated with neurological complications in HFMD infections. ${ }^{(7)}$ In addition, our finding that CSF pleocytosis was only detected in patients who developed neurological manifestations suggests that a spinal tap can be both diagnostic and prognostic for the development of neurological manifestations in these children. While we found that poor oral intake was associated with children who did not develop any neurological manifestations, this may not be clinically significant. We did not identify other non-neurological symptoms that were associated with the development of neurological manifestations in our study.

The EV71 serotype was isolated from only $13.0 \%$ of our tested samples from CSF, throat or rectal swabs, indicating that EV71 remains a relatively infrequent serotype locally. The EV71 serotype has been known to be more frequently associated with neurological complications and chronic neurological sequelae. One study from China showed that neurological manifestations were found in $73 \%$ of EV71-infected children and $27.9 \%$ of CA16-infected children. ${ }^{(10)}$ However, in our study, only $5.3 \%$ of children who developed neurological manifestations tested positive for the EV71 serotype, and $66.7 \%$ (two out of three children) who tested positive for EV71 serotype did not develop any neurological manifestations. Although the numbers in our study were low, these findings emphasised the variability of neurological involvement with the EV71 strain despite its known virulence. It also highlights the occurrence of mild EV71 disease with no neurological manifestations.

Our results suggest that enterovirus infections can be associated with significant long-term neurological sequelae, as $16.7 \%$ of our patients with neurological morbidities developed sequelae such as epilepsy, chronic myoclonic jerks, behavioural changes or cognitive decline, developmental delay and autonomic dysfunction. The rate of long-term neurological sequelae in children with enterovirus infections in our study was comparable to that of viral infections of other aetiologies. For instance, in a study looking at neurological complications associated with varicella zoster infections, residual neurological sequelae at one year occurred in nine of 39 children (23.1\%) with follow-up data. ${ }^{(19)}$

The small number of patients in this cohort should be recognised as a major limitation of the study. A larger number of patients may be necessary to verify significant results in future cohort studies. In addition, only EV71 serotype identification was carried out. It would be useful to include the identification of other viral serotypes in future studies, so that their association with the range of clinical manifestations and comorbidities can be reviewed. This would provide us with better accuracy in predicting disease severity in children from our local population who are infected with enterovirus infections, based on known viral serotypes involved in local disease outbreaks. Furthermore, with new virulent strains affecting regions of North America recently, ${ }^{(20-22)}$ it would be important to establish the identification of enterovirus strains as part of regular surveillance in Singapore.

In conclusion, we identified a broad spectrum of neurological manifestations, including some unusual neurological disease presentations, in Singaporean children with enterovirus infections. However, the incidence of long-term neurological sequelae remained low, as observed in our study. Nonetheless, some of these neurological morbidities were severe and, therefore, careful evaluation of children with neurological involvement is necessary. Further work needs to be done so that we can have a better understanding of the wide spectrum of disease presentations and clinical morbidities associated with enterovirus infections in the paediatric population. 


\section{REFERENCES}

1. Chan KP, Goh KT, Chong $\mathrm{CY}$, et al. Epidemic hand, foot and mouth disease caused by human enterovirus 71, Singapore. Emerg Infect Dis 2003; 9:78-85.

2. Lum LC, Wong KT, Lam SK, et al. Fatal enterovirus 71 encephalomyelitis. J Pediatr 1998; 133:795-8.

3. Ho M, Chen ER, Hsu KH, et al. An epidemic of enterovirus 71 infection in Taiwan. Taiwan Enterovirus Epidemic Working Group. N Engl J Med 1999; 341:929-35.

4. Ryu WS, Kang B, Hong J, et al. Enterovirus 71 infection with central nervous system involvement, South Korea. Emerg Infect Dis 2010; 16:1 764-6.

5. Tu PV, Thao NT, Perera D, et al. Epidemiologic and virologic investigation of hand, foot, and mouth disease, southern Vietnam, 2005. Emerg Infect Dis 2005; 13:1733-41.

6. Mao LX, Wu B, Bao WX, et al. Epidemiology of hand, foot, and mouth disease and genotype characterization of Enterovirus 71 in Jiangsu, China. J Clin Virol 2010; 49:100-4.

7. Kim SJ, Kim JH, Kang JH, et al; Enteroviruses Complications Working Group. Risk factors for neurologic complications of hand, foot and mouth disease in the Republic of Korea, 2009. J Korean Med Sci 2013; 28:120-7.

8. Lu HK, Lin TY, Hsia SH, et al. Prognostic implications of myoclonic jerk in children with enterovirus infection. J Microbiol Immunol Infect 2004; 37:82-7.

9. Wu Y, Yeo A, Phoon MC, et al. The largest outbreak of hand; foot and mouth disease in Singapore in 2008: the role of enterovirus 71 and coxsackievirus A strains. Int J Infect Dis 2010; 14:e1076-81.

10. Xu W, Liu CF, Yan L, et al. Distribution of enteroviruses in hospitalized children with hand, foot and mouth disease and relationship between pathogens and nervous system complications. Virol J 2012; 9:8.

11. Chen SP, Huang YC, Li WC, et al. Comparison of clinical features between coxsackievirus A2 and enterovirus 71 during the enterovirus outbreak in Taiwan, 2008: a children's hospital experience. J Microbiol Immunol Infect 2010; 43:99-104.
12. Wang SM, Ho TS, Lin HC, et al. Reemerging of enterovirus 71 in Taiwan: the age impact on disease severity. Eur J Clin Microbiol Infect Dis 2012; $31: 1219-24$

13. Shah VA, Chong CY, Chan KP, Ng W, Ling AE. Clinical characteristics of an outbreak of hand, foot and mouth disease in Singapore. Ann Acad Med Singapore 2003; 32:381-7.

14. Ang LW, Koh BK, Chan KP, et al. Epidemiology and control of hand, foot and mouth disease in Singapore, 2001-2007. Ann Acad Med Singapore 2009; 38:106-12.

15. Chong $\mathrm{CY}$, Chan KP, Shah VA, et al. Hand, foot and mouth disease in Singapore: a comparison of fatal and non-fatal cases. Acta Paediatr 2003; 92:1163-9.

16. Graham AK, Murdoch DR. Association between cerebrospinal fluid pleocytosis and enteroviral meningitis. J Clin Microbiol 2005; 43:1491.

17. Mulford WS, Buller RS, Arens MQ, Storch GA. Correlation of cerebrospinal fluid (CSF) cell counts and elevated CSF protein levels with enterovirus reverse transcription-PCR results in pediatric and adult patients. J Clin Microbiol 2004; 42:4199-203

18. Pérez-Vélez CM, Anderson MS, Robinson CC, et al. Outbreak of neurological enterovirus 71 disease: a diagnostic challenge. Clin Infect Dis 2007; 45:950-7.

19. Science M, MacGregor D, Richardson SE, et al. Central nervous system complications of varicella-zoster virus. J Pediatr 2014; 165:779-85.

20. Maloney JA, Mirsky DM, Messacar K, et al. MRI findings in children with acute flaccid paralysis and cranial nerve dysfunction occurring during the 2014 enterovirus D68 outbreak. AJNR Am J Neuroradiol 2015; 36:245-50.

21. Shaw J, Welch TR, Milstone AM. The role of syndromic surveillance in directing the public health response to the enterovirus D68 epidemic. JAMA Pediatr 2014; 168:981-2.

22. Pastula DM, Aliabadi N, Haynes AK, et al; Centers for Disease Control and Prevention (CDC). Acute neurologic illness of unknown etiology in children - Colorado, August-September 2014. MMWR Morb Mortal Wkly Rep 2014; 63:901-2. 